\title{
Juvenile psammomatoid ossifying fibroma with fluid-fluid levels: an unusual presentation
}

\author{
Linda Kalliath ${ }^{1 *}$, D. Karthikeyan', Nataraj Pillai ${ }^{2}$, Deepthi Padmanabhan², Partheeban Balasundaram ${ }^{3}$ and \\ Gokul Kripesh ${ }^{3}$
}

\begin{abstract}
Background: Juvenile ossifying fibroma is an uncommon benign fibro-osseous tumor commonly involving the craniofacial skeleton of young patients with locally aggressive behavior and a high recurrence rate. Depending on the site of involvement it can present clinically as nasal obstruction, facial swelling, or proptosis.

Case presentation: Here we present a case of juvenile ossifying fibroma with an aneurysmal bone cyst component in the left nasal cavity in a 15-year-old boy who presented with gradually progressing left-sided nasal obstruction. Imaging findings were consistent with juvenile ossifying fibroma. Endoscopic resection of the tumor was done, and histopathological examination revealed it to be a psammomatoid variant of juvenile ossifying fibroma. Here we discuss its imaging findings, differential diagnosis, treatment options, and histopathological features.
\end{abstract}

Conclusion: Despite being a slow-growing benign tumor, early diagnosis and treatment are necessary due to its locally aggressive nature and invasion of adjacent structures. Complete surgical resection is the mainstay of treatment because of the high recurrence rate.

Keywords: Juvenile psammomatoid ossifying fibroma, Fibro-osseous lesions, Aneurysmal bone cyst, Fluid-fluid levels

\section{Background}

Juvenile ossifying fibroma is a unique entity primarily seen in children and adolescents. It is a benign fibroosseous tumor commonly seen in the sinonasal region. It differs from the conventional ossifying fibroma by the age of presentation, site of involvement, its locally aggressive nature, and high recurrence rate. Depending on the site of involvement it can present clinically as nasal obstruction, facial swelling or proptosis Two histopathological variants have been described: namely psammommatoid and the trabecular type, each having distinctive radiological and histopathological features. Aneurysmal bone cyst formation and areas of hemorrhage are seen as secondary changes associated with juvenile ossifying fibroma. The

\footnotetext{
${ }^{*}$ Correspondence: lindakalliath@gmail.com

1 Department of Radiology, SRM Institutes for Medical Science,

Vadapalani, Chennai, India

Full list of author information is available at the end of the article
}

differential diagnosis for fibro-osseous lesions includes fibrous dysplasia, cemento-osseous dysplasia, and ossifying fibroma.

\section{Case presentation}

A 15-year-old boy presented with gradually progressing left-sided nasal obstruction for 2 years. There was no history of epistaxis, rhinorrhea, headache, or blurred vision. Nasal examination revealed a single pink polypoidal mass lesion filling the left nasal cavity (Fig. 1) with deviated nasal septum towards the right. On probing it was firm in consistency. Initially, the patient was evaluated elsewhere, where they attempted an endoscopic biopsy which was abandoned, as a hemorrhagic aspirate of $17 \mathrm{cc}$ was obtained from the left nasal mass. The patient was referred to our center for further management.

Unenhanced computed tomography $(\mathrm{CT})$ scan revealed an expansile mass lesion measuring $\sim 4.6 \times 4.2 \times 4.0 \mathrm{~cm}$ with central heterogeneous density and sclerosed 


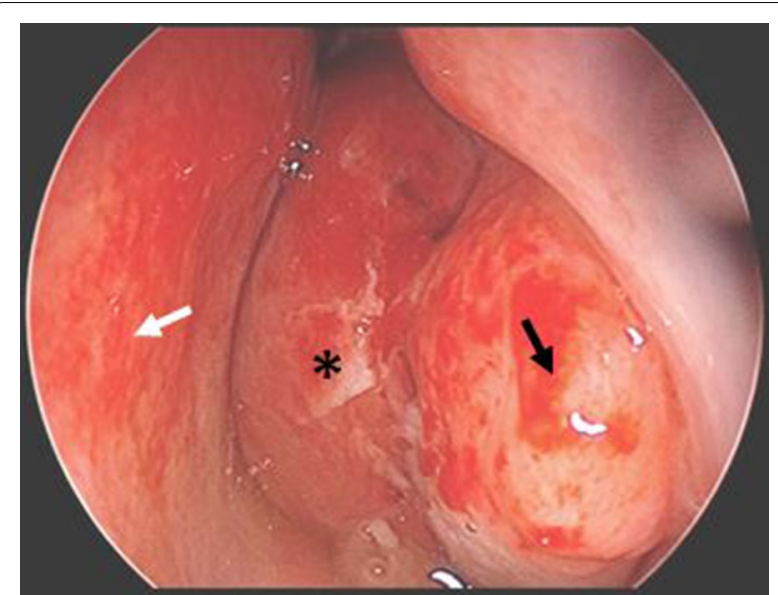

Fig. 1 Endoscopic view displaying pinkish polypoidal mass lesion $\left(^{*}\right)$ in the left nasal cavity. Note the Nasal septum (white arrow) and Inferior turbinate (black arrow)

peripheral mantle in the left nasal cavity (Fig. 2). Remodeling of the adjacent maxillary sinus, ethmoidal sinuses and nasal septum adjacent to the lesion with rarefaction of sinus walls and nasal septum were noted on the left side. The lesion was displacing and remodeling the left inferior turbinate.

Subsequent multisequence multiplanar magnetic resonance imaging (MRI) revealed, the central region with T2 hypointense/T1 isointense areas, fluid-fluid levels (Fig. 3), T1 hyperintense areas nonsuppressed in fat sat images and blooming in gradient-echo sequence. The peripheral mantle appeared T2 hypointense and T1 isointense which was corresponding to the hyperdense area in CT. Heterogeneous enhancement of the lesion was noted in the contrast study (Fig. 4). Fluid intensity was noted within the left maxillary and ethmoidal sinuses and left chamber of sphenoid sinus, likely being inspissated secretions. Mild displacement of intact inferomedial wall of the posterior aspect of left orbit by the lesion was also seen. No significant mass effect within the orbital contents was observed. No obvious intraorbital or intracranial extension of the lesion was evident. From the imaging findings, a diagnosis of juvenile ossifying fibroma with secondary aneurysmal bone cyst formation was made.

The patient underwent surgical excision of the left nasal mass lesion. On macroscopic examination, the tissue was received in piece-meal, firm, grey-white to tan-brown in color and gritty in consistency. Microscopic examination revealed sinus mucosa with a fibro-osseous tumor displaying pushing borders. The tumor was composed of a moderately cellular fibrous stroma with intervening ossicles resembling psammoma bodies (Fig. 5). Bands of osteoid and immature bony trabeculae surrounded by plump osteoblasts were seen. There was no evidence of brisk mitotic activity or nuclear atypia. Focal cystic changes were seen, which is consistent with the aneurysmal cyst component on imaging. A diagnosis of juvenile psammomatoid ossifying fibroma was made. Post-operative follow-up reveals no significant residual lesions and satisfactory recovery noted.

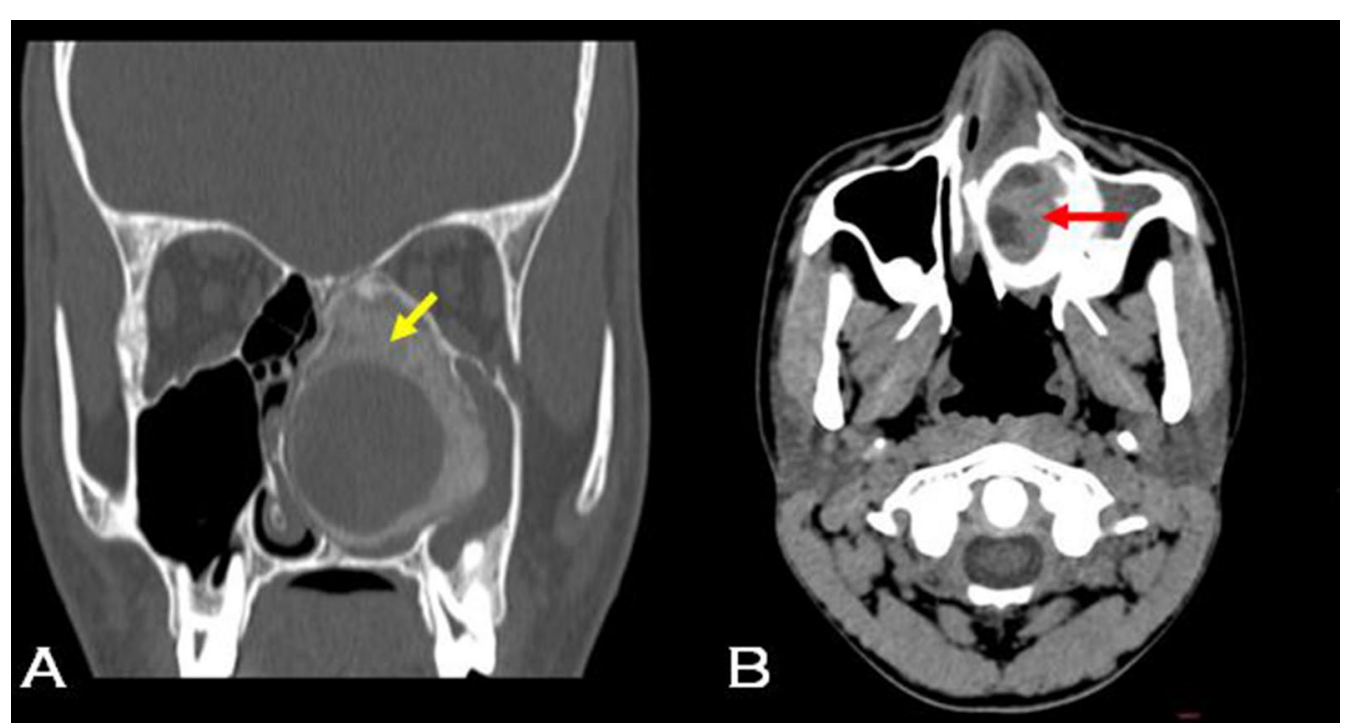

Fig. 2 Coronal unenhanced CT scan (A) revealing well-demarcated expansile lesion with peripheral sclerotic rind (yellow arrow) and (B) showing heterogeneous density central fibrous core (red arrow) 


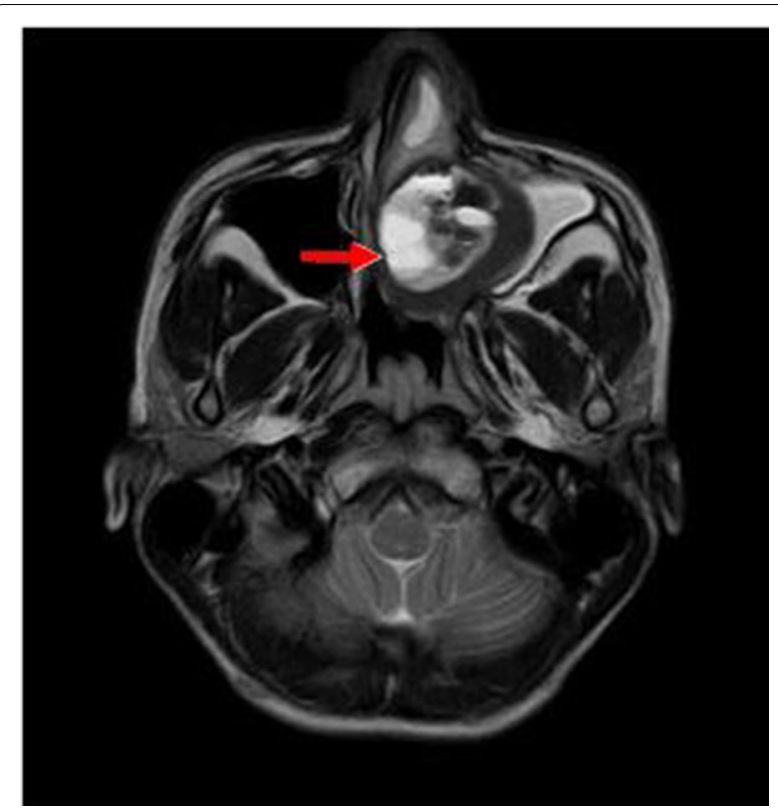

Fig. 3 Axial T2 weighted MRI image showing central hyper and isointense areas with fluid-fluid levels (red arrow)

\section{Discussion}

Ossifying fibroma is a rare fibro-osseous tumor composed of fibrous tissue and mature bone. It is subdivided into conventional and juvenile subtypes. Juvenile ossifying fibroma is an uncommon variant of ossifying fibroma that behaves in a more aggressive pattern than the conventional type [1]. The incidence of juvenile ossifying fibroma is higher in children under the age of 15 years. It commonly occurs in the craniofacial skeleton, $90 \%$ in the orbit and paranasal sinuses; whereas the classical ossifying fibroma occurs commonly in the mandible. Hence the age of the patient, site of involvement, high recurrence tendency, and aggressive behavior helps to differentiate juvenile ossifying fibroma from conventional ossifying fibroma [2]. The clinical presentation depends on the site of involvement and it can be epistaxis, nasal obstruction, facial swelling, or proptosis [1].

Juvenile ossifying fibroma can be subdivided into two histopathological patterns: Trabecular type and psammomatoid type. Trabecular type is seen in a younger age group (8-12 years) than the psammomatoid variant (16-33 years) [3]. Internal calcified components are characteristic of trabecular type whereas psammomatoid variant usually presents as homogeneous ground-glass pattern [4]. Psammomatoid juvenile ossifying fibroma results from increased production of myxofibrous cellular stroma [1]. Aneurysmal bone cyst formation is seen in association with psammomatoid juvenile ossifying fibroma and it is due to increased formation of osteoclast giant cells which causes expansion and cyst formation within the myxofibrous stroma. Development of an aneurysmal bone cyst is seen commonly in younger patients (1st and 2nd decades) with an aggressive nature and high recurrence rate [5]. Areas of hemorrhage and aneurysmal bone cyst formation are common secondary changes associated with juvenile ossifying fibroma

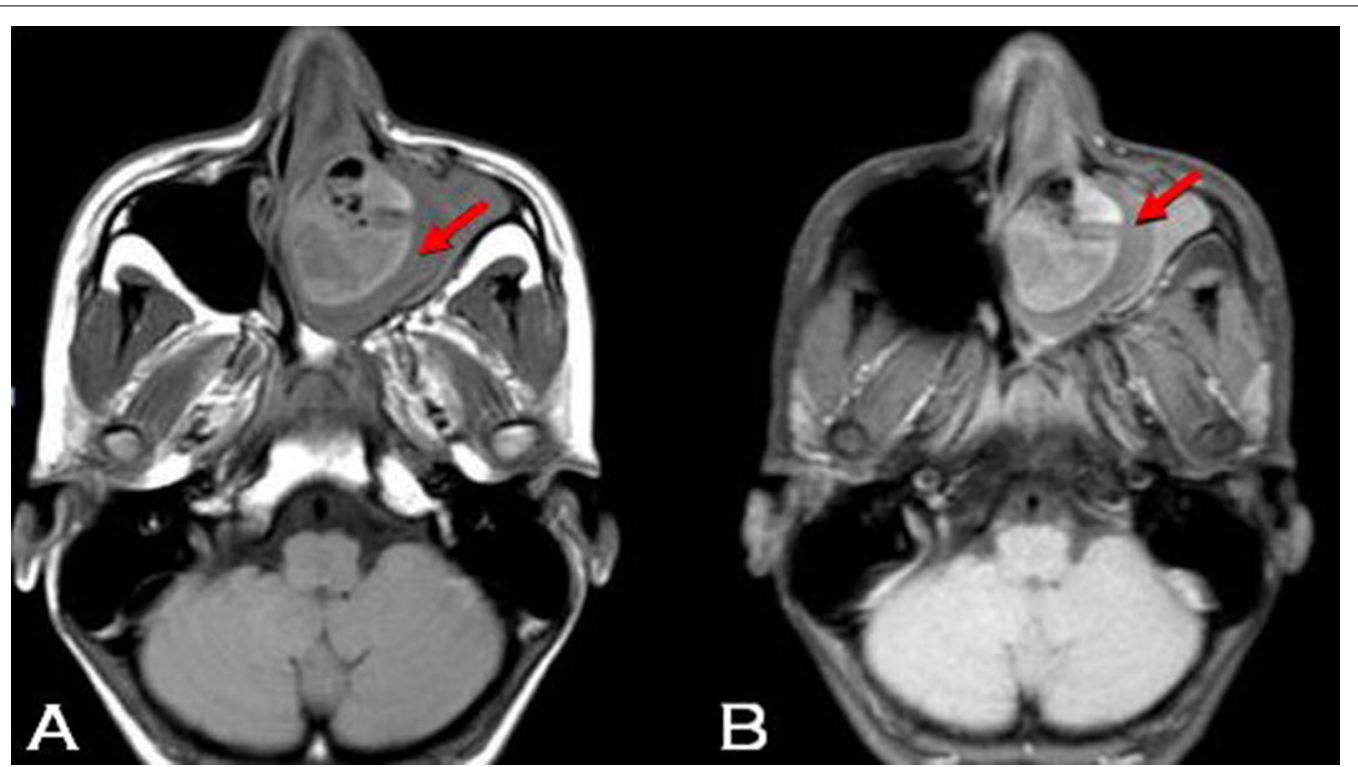

Fig. 4 Axial T1 weighted MRI image revealing $(\mathbf{A})$ isointense peripheral mantle (red arrow) and heterogeneous central core, which is seen enhancing in post-contrast fat-suppressed T1 weighted image (B) 


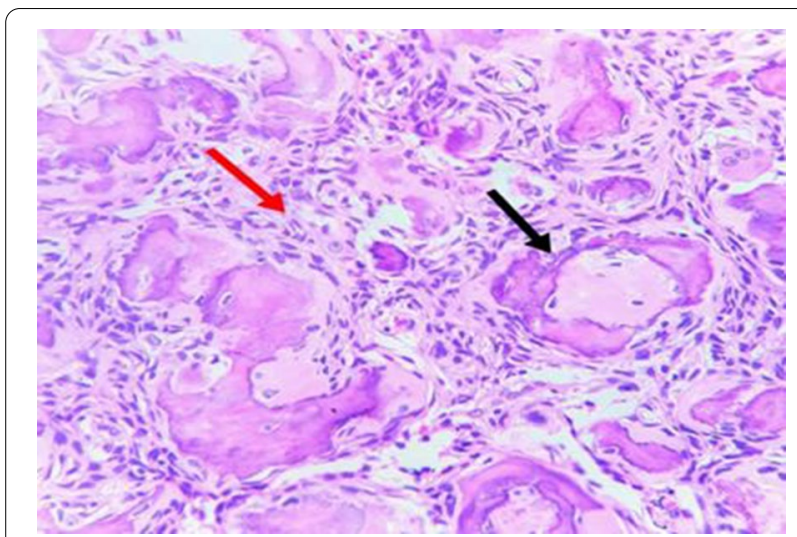

Fig. $540 \times$ magnification of H\&E stained slide displaying psammoma bodies-like ossicles (marked with black arrow), set in a moderately cellular spindle cell fibrous stroma (marked with red arrow)

as in our case [1]. In a study by Makek, the incidence of aneurysmal bone cyst formation in juvenile ossifying fibroma is $4.3 \%$ [6]. Juvenile ossifying fibroma can be radiographically divided into 3 stages. Initial stage/Stage I-It appears as a well-defined radiolucent lesion. Mixedstage/Stage II-Radiolucent lesion with areas of internal calcification. Mature stage/Stage III-Completely radioopaque mass[1]. On CT it appears as a well-demarcated lesion with a sclerotic rim and less dense fibrous central core. Three patterns have been described in CT. 1st pattern-Radiolucent central core with a thick outer mantle. 2nd pattern-Ground glass mural nodule. 3rd patternHomogeneous solid radiodense lesion [7]. The lesion can expand and involve multiple anatomical spaces resulting in ballooning and bowing of involved bones. Cystic areas can appear which may contain blood and represent secondary aneurysmal bone cyst formation [8]. On MRI the sclerotic rim appears $\mathrm{T} 1$ isointense and $\mathrm{T} 2$ hypointense which shows enhancement on contrast administration [7]. The solid component of the lesion appears isointense to muscle on T1 images, iso-hypointense on T2 images. On contrast administration, heterogeneous/diffuse enhancement of the solid component and peripheral enhancement of cystic component with layering of blood products are seen within [8].

Differential diagnosis includes fibrous dysplasia, cemento osseous dysplasia, aneurysmal bone cyst, mucocele, and osteoblastoma. Fibrous dysplasia is the most important differential diagnosis of juvenile ossifying fibroma [8]. In contrast to juvenile ossifying fibroma, fibrous dysplasia typically shows a more homogeneous internal structure with ill-defined margins which merge with the surrounding bone causing bony expansion and maintains the shape/configuration of the involved bone [9]. Juvenile ossifying fibroma appears as a well-defined radiolucent lesion with a thin rim of surrounding sclerotic area and the bony expansion is more of a spherical configuration [4]. Another common differential is cemento osseous dysplasia. It is commonly seen in tooth-bearing areas especially in the alveolar process of the maxilla and mandible, which confirms the odontogenic origin of the lesion [10]. It does not cause bony expansion [7]. An aneurysmal bone cyst is not common in the sinonasal region. Punctate areas of calcification are also an uncommon feature. The characteristic imaging finding is fluid-fluid levels on MRI. It is commonly seen as an association or reactive change in preexisting bone lesions. Osteoblastomas are uncommon in the skull and facial bones. At imaging, it is difficult to differentiate it from the juvenile ossifying fibroma. Microscopically presence of abundant osteoid elements and atypical mitosis helps in the differentiation of osteoblastoma from the juvenile ossifying fibroma [8]. Mucoceles are peripherally enhancing expansile lesions of sinuses with smooth remodeling of bone and extension to adjacent tissue, secondary to ostial obstruction. Site of origin and enhancement characteristics helps to differentiate it from juvenile ossifying fibroma [9].

Complete surgical excision rather than conservative curettage is the preferred line of treatment in both subtypes of juvenile ossifying fibroma [4]. With incomplete resection, the possibility of recurrence is higher and is more aggressive than primary which is seen in a period of 6 months to 19 years following surgery, hence it requires long-term follow-up [2]. Depending on the extent of disease and invasion, an open surgical approach might be necessary rather than an endoscopic approach for complete resection [7]. Radiotherapy is contraindicated, as the tumor is radioresistant, with a high risk of malignant transformation, and late radiotherapy-related harmful effects in children are seen [11]. Due to the high recurrence rate (30-50\%) and aggressive behavior of the lesion, juvenile ossifying fibroma should be treated as a locally aggressive neoplasm [2].

\section{Conclusion}

Juvenile ossifying fibroma despite being benign and slowgrowing behaves in a locally aggressive manner with the invasion of adjacent anatomical structures. The presence of a well-circumscribed lesion with a sclerotic rim and central less dense fibrous core and enhancement of the solid component should raise the suspicion of juvenile ossifying fibroma. Complete surgical resection is the mainstay of treatment because of the high risk of local recurrence which is found to be more aggressive than the primary. 
Abbreviations

$\mathrm{CT}$ : Computed tomography; MRI: Magnetic resonance imaging.

\section{Acknowledgements}

Not applicable.

\section{Authors' contributions}

LK collected the data and wrote the manuscript; KD helped in editing and supervising the paper; NP and DP provided the patient's clinical data and images; PB and GK responsible for the curation of gross pathology and histological images. All authors have read and approved the final manuscript.

\section{Funding}

Not applicable.

\section{Availability of data and materials}

All data is available based on a reasonable request.

\section{Declarations}

Ethics approval and consent to participate

Not applicable.

\section{Consent for publication}

Formal informed consent was obtained from the patient's parent via telephone. Written consent could not be obtained as the patient's parents did not want to return for follow-up due to concern for COVID and associated restrictions. The patient and his family also live away from our institution without the ease of transportation.

\section{Competing interests}

The authors declare that they have no competing interests.

\section{Author details}

'Department of Radiology, SRM Institutes for Medical Science, Vadapalani, Chennai, India. ${ }^{2}$ Department of ENT, SRM Institutes for Medical Science, Vadapalani, Chennai, India. ${ }^{3}$ Department of Pathology, SRM Institutes for Medical Science, Vadapalani, Chennai, India.

Received: 9 June 2021 Accepted: 26 July 2021

Published online: 03 August 2021

\section{References}

1. Khan M, Ramachandra VK, Rajguru P (2014) A case report on juvenile ossifying fibroma of the mandible. J Indian Acad Oral Med Radiol 26(2):213. https://doi.org/10.4103/0972-1363.143706

2. Reddy AV, Reddy KR, Prakash AR (2014) Juvenile ossifying fibroma with aneurysamal bone cyst: a case report. J Clin Diagn Res JCDR 8(10):ZD01. https://doi.org/10.7860/JCDR/2014/8909.4952

3. Owosho AA, Hughes MA, Prasad JL, Potluri A, Branstetter B (2014) Psammomatoid and trabecular juvenile ossifying fibroma: two distinct radiologic entities. Oral Surg Oral Med Oral Pathol Oral Radiol 118(6):732-738. https://doi.org/10.1016/j.oooo.2014.09.010

4. Owosho AA, Hughes MA, Prasad JL, Potluri A, Costello BJ, Branstetter BF IV (2015) Is computed tomography an adequate imaging modality for the evaluation of juvenile ossifying fibroma? A comparison of 2 imaging modalities (computed tomography and magnetic resonance imaging). J Oral Maxillofac Surg 73(7):1304-1313. https://doi.org/10.1016/j.joms. 2015.01.013

5. Gotmare SS, Tamgadge A, Tamgadge S, Kesarkar KS (2017) Recurrent psammomatoid juvenile ossifying fibroma with aneurysmal bone cyst: an unusual case presentation. Iran J Med Sci 42(6):603

6. Makek M (1983) Clinical pathology of fibro-osteo-cemental lesions in the cranio-facial and jaw bones. Karger Publishers, Basel

7. Nguyen S, Hamel MA, Chénard-Roy J, Corriveau MN, Nadeau S (2019) Juvenile psammomatoid ossifying fibroma: a radiolucent lesion to suspect preoperatively. Radiol Case Rep 14(8):1014-1020. https://doi.org/10. 1016/j.radcr.2019.05.017

8. Chang HJ, Donahue JE, Sciandra KT, Evangelista PT (2009) Juvenile ossifying fibroma of the calvaria. Radiographics 29(4):1195-1199. https://doi. org/10.1148/rg.294085240

9. Kendi AT, Kara S, Altinok D, Keskil S (2003) Sinonasal ossifying fibroma with fluid-fluid levels on MR images. Am J Neuroradiol 24(8):1639-1641

10. Kaur T (2021) Fibro-osseous lesions in the maxillofacial region. Oral Maxillofac Surg Clin 615-28

11. Linhares P, Pires E, Carvalho B, Vaz R (2011) Juvenile psammomatoid ossifying fibroma of the orbit and paranasal sinuses. A case report. Acta Neurochir 153(10):1983-1988. https://doi.org/10.1007/s00701-011-1115-1

\section{Publisher's Note}

Springer Nature remains neutral with regard to jurisdictional claims in published maps and institutional affiliations.

\section{Submit your manuscript to a SpringerOpen ${ }^{\circ}$ journal and benefit from:}

- Convenient online submission

- Rigorous peer review

- Open access: articles freely available online

- High visibility within the field

- Retaining the copyright to your article

Submit your next manuscript at $\boldsymbol{\nabla}$ springeropen.com 